Produto \& Produção, vol. 13 n. 1, p. 01-15, fev. 2012

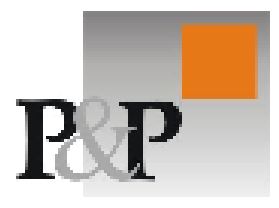

\title{
Caracterização de um modelo mínimo para o PDS por meio de uma revisão sistemática de literatura.
}

Recebido em 03/11/2011. Aceito em 15/02/2012.

\section{Patricia Flores Magnago}

Departamento de Engenharia de Produção e Transportes - DEPROT/UFRGS

pp fm@hotmail.com

\section{Márcia Elisa Echeveste \\ Departamento de Estatística_IMA/UFRGS \\ echeveste@producao.ufrgs.br}

Este estudo tem o objetivo de caracterizar um modelo mínimo para o Processo de Desenvolvimento de Serviços (PDS). Para isso, contou com a identificação das características e das fases comuns dos principais modelos de PDS existentes na literatura. Os modelos de PDS existentes foram escolhidos utilizando-se de uma revisão sistemática da literatura, que incluiu um estudo comparativo entre catorze modelos. O modelo mínimo proposto apresenta nove fases e, detalha em profundidade, oito atividades-chave inseridas em duas das fases. O propósito de caracterização de um modelo mínimo visa padronizar a linguagem, consolidar atividades essenciais e facilitar o entendimento do PDS; servindo de apoio para as empresas que visam simplificar a sistematização do desenvolvimento de seus serviços ou orientar as empresas que não possuem estrutura para este processo.

Palavras chave: projeto de serviços; revisão sistemática; modelos de PDS.

This study aims at characterizing a minimal model for Service Development Process (SDP). To this end, this study relied on the identification of common features and phases of the main models of SDP in the literature. The SDP existing models were chosen using a systematic literature review, which included a comparative study of fourteen models. The proposed minimal model presents nine stages and details, in depth, eight key activities included in two of the phases. The purpose of the characterization of a minimal model is to standardize the language, consolidate essential activities and facilitate the understanding of SDP, serving as support for companies that aim at simplifying the systematic development of their services or to provide guidance for companies that have no structure for this process.

Keywords: service project; systematic review; models of SDP. 


\section{INTRODUÇÃO}

Os serviços tem sido responsáveis pela ocupação da mão-de-obra e pela geração de riqueza em muitos países. Isso reflete a tendência do aumento da participação dos serviços no Produto Interno Bruto (PIB). Contudo, muitos serviços estão envolvidos em ambientes de acirrada competitividade e em mercados em constante estado de transformação (D'AVENI, 1994). As empresas passaram, então, a visar à estratégia de adoção de novos serviços e a de melhoria contínua dos serviços já existentes, buscando assegurar a qualidade desses aos seus clientes. Dada esta dinâmica, como os novos serviços são desenvolvidos e lançados está se tornando cada vez mais importante para garantir o bom desempenho e a vantagem frente à concorrência (FITZSIMMONS; FITZSIMMONS, 2005) .

A natureza dos serviços, centrada na falta de tangibilidade, está entre as causas das dificuldades de garantir a sua qualidade e projetá-los (LEVITT, 1981). A fim de tornar o serviço mais visível e gerenciável é importante a elaboração de um adequado projeto de serviços, o qual pode se constituir um elemento chave para assegurar a qualidade depois de lançados (MA et al, 2002). Conforme Shostack (1984), as falhas em serviços decorrem da incompetência humana e da falta de um método sistemático para projeto e controle, sendo o desenvolvimento de um novo serviço caracterizado por tentativa e erro. Uma linguagem sistemática estruturada é capaz de impulsionar a capacidade das empresas em desenvolvê-los (MELLO et al 2005). O projeto de serviços acontece dentro do Processo de Desenvolvimento de Serviços (PDS).

Diversos autores na literatura buscaram desenvolver modelos sistemáticos de PDS visando garantir o sucesso neste processo (COOPER; DE BRENTANI, 1991). Observando o reconhecido Modelo de Gaps da Qualidade em Serviços de Parasuraman et al (1985), a aplicação dos conceitos de projeto e processo de serviço contribui na redução das lacunas existentes entre o serviço esperado pelo cliente, a percepção da gerência sobre essas expectativas, as especificações do serviço e a prestação do serviço em si. O que nota-se, de fato, é que as empresas ainda não possuem um foco estratégico no PDS e ainda faltam competências e estruturas apropriadas para dar suporte ao processo (EASINGWOOD, 1986; EDGETT, 1993; MARTIN; HORNE, 1993; SCHEUING; JOHNSON, 1989).

O PDS permanece entre os temas menos estudados na literatura de gerenciamento de serviços, constituindo-se um paradoxo na área, uma vez que existe extensa literatura de modelos para o processo de desenvolvimento de produtos (PDP), especialmente nos últimos anos (MENOR et al, 2002). Behara e Chase (1993) enfatizam o paradoxo afirmando que "se nós projetarmos os carros da forma que os serviços são projetados, eles provavelmente viriam com um eixo e cinco rodas". Page e Schirr (2008) realizaram uma revisão das publicações internacionais de PDP entre os anos de 1989 e 2004, e constataram que menos de $3 \%$ dos 815 artigos avaliados continham uma abordagem conjunta ao PDS. Já as publicações, exclusivas sobre o PDS, dobraram de volume entre os anos de 1997 a 2002, porém ainda existem muitas oportunidades de estudos, entre elas a análise da capacidade de inovação no PDS (JONG; VERMEULEN, 2003).

Menor et al (2002) consolidaram catorze oportunidades de estudos no que diz respeito ao PDS. Entre elas está a compreensão das fases e atividades do PDS e o levantamento das características de um PDS executado com sucesso. Os autores, embasados na literatura, apresentam três questões de pesquisa que podem ser 
levantadas para esta oportunidade, são elas: existem estágios comuns no PDS e eles são sempre necessários? (BOWERS, 1985); quão formalizado o PDS precisa ser? (DESZCA et al, 1999) e quais atividades necessárias na execução de um PDS de sucesso? (JOHNSON et al, 2000). Estas questões buscam ser tratadas por autores que criaram modelos sistemáticos para o PDS.

Neste sentido, o objetivo deste estudo é identificar as características e fases comuns dos diversos modelos de PDS existentes na literatura, levantados por meio de uma revisão sistemática, para compor um modelo mínimo para o PDS, com a finalidade de tornar esse processo mais fácil de ser gerenciado e repetido pelas empresas de serviço. As contribuições deste estudo estão apresentadas em cinco seções. Após esta introdução, a segunda seção traz as considerações referentes ao método de pesquisa. Em seguida, na terceira seção, é apresentada a revisão de literatura, contando com uma análise comparativa entre os modelos estudados. $\mathrm{Na}$ quarta seção são apresentados os resultados, abordando a estrutura faseada do modelo mínimo de PDS. Por fim, a última seção expõe as conclusões do trabalho, também descrevendo sugestões para trabalhos futuros.

\section{MÉTODO}

Este estudo é uma pesquisa de natureza aplicada e com objetivos de caráter exploratório; uma vez que faz um levantamento qualitativo das publicações quanto ao PDS. O levantamento das publicações foi realizado utilizando o método de revisão sistemática da literatura.

A revisão sistemática é uma forma de pesquisa que utiliza a literatura sobre um determinado tema como fonte primária de dados. Portanto, a revisão é dependente da qualidade das fontes primárias e constitui-se de um tipo de investigação que disponibiliza um resumo das evidências relacionadas a uma estratégia de intervenção de interesse. A intervenção, ou seja, o objetivo de interesse é definido logo ao início do método, que se desenvolve por meio de etapas que permitem a busca, apreciação crítica e síntese da informação selecionada. Este método é indicado para integrar informações de um conjunto de estudos realizados separadamente que podem apresentar resultados conflitantes e/ou coincidentes (LINDE; WILLICH, 2003).

A revisão sistemática foi escolhida para este estudo, principalmente, por permitir incorporar as contribuições de um volume maior de resultados relevantes, ao invés de limitar a conclusão apenas sobre alguns autores, possibilitando a avaliação da consistência e capacidade de generalização dos resultados (AKOBENG, 2005). Na Figura 1 são apresentadas as etapas que compõem a revisão sistemática proposta por Sampaio e Mancini (2007) advindas de adaptações aos trabalhos de Domholdt (2005), Law e Philp (2002) e Magee (1998). Também na Figura 1, para cada etapa da revisão, são explicadas como foi a operacionalização para este estudo.

\begin{tabular}{l|l}
\hline \multicolumn{1}{c|}{ Etapas } & \multicolumn{1}{c}{ Operacionalização para o estudo } \\
\hline $\begin{array}{l}\text { Definir a pergunta } \\
\text { científica; }\end{array}$ & $\begin{array}{l}\text { Como agrupar as características comuns dos modelos de PDS em um modelo } \\
\text { simplificado? }\end{array}$ \\
\hline $\begin{array}{l}\text { Identificar as } \\
\text { bases de dados, }\end{array}$ & $\begin{array}{l}\text { Alguns dos periódicos que serviram para consulta, indicados pela busca na base de dados } \\
\text { do Google Scholar, foram publicados em Journals como: o Harvard Business Review, o }\end{array}$ \\
\hline
\end{tabular}




\begin{tabular}{|c|c|}
\hline $\begin{array}{l}\text { definir palavras- } \\
\text { chaves e } \\
\text { estratégias de } \\
\text { busca; }\end{array}$ & $\begin{array}{l}\text { European Management e o Journal of Services Marketing. Além dos periódicos, foram } \\
\text { realizadas consultas a documentos da normatização alemã, DIN (2008), centros de } \\
\text { pesquisa em serviços, Fraunhofer (2008), e trabalhos de teses nacionais, como Mello } \\
\text { (2005). Para a pesquisa nas bases de dados foram utilizadas as palavras-chaves: New } \\
\text { Service Development e Service Design. Neste trabalho foi empregada como estratégia de } \\
\text { busca as 'referências cruzadas' entre publicações, portanto permitindo encontrar } \\
\text { trabalhos mais antigos sobre o tema. }\end{array}$ \\
\hline $\begin{array}{l}\text { Estabelecer } \\
\text { critérios para a } \\
\text { seleção dos } \\
\text { artigos; }\end{array}$ & $\begin{array}{l}\text { Os trabalhos selecionados para a o estudo atenderam aos seguintes critérios de } \\
\text { qualificação: (i) existência de modelos de PDS faseados, que fossem também claramente } \\
\text { identificados e caracterizados pelos seus autores; (ii) trabalhos que representem } \\
\text { unidades de análise de diferentes países; (iii) trabalhos representativos de diferentes } \\
\text { períodos de tempo; (iv) trabalhos de distintos grupos de pesquisadores; e (v) trabalhos } \\
\text { com modelos de PDS desenvolvidos para diversas áreas (setores) de serviços. }\end{array}$ \\
\hline $\begin{array}{l}\text { Conduzir buscas } \\
\text { nas bases de } \\
\text { dados e comparar } \\
\text { buscas dos } \\
\text { examinadores; }\end{array}$ & $\begin{array}{l}\text { As buscas aos modelos de PDS foram realizadas no período de um mês. Não houve } \\
\text { comparação entre as buscas de mais de um examinador, pois os dois examinadores } \\
\text { trabalharam em conjunto para a composição da base de periódicos, organizados no } \\
\text { software Mendeley, avaliando sempre o número de citações, como fator da identificação } \\
\text { de relevância dos trabalhos a serem lidos. }\end{array}$ \\
\hline $\begin{array}{l}\text { Aplicar critérios } \\
\text { na seleção dos } \\
\text { artigos e justificar } \\
\text { exclusões; }\end{array}$ & $\begin{array}{l}\text { Foram levantados dezesseis trabalhos, sendo selecionados catorze para a comparação. As } \\
\text { exclusões de dois modelos são justificadas por serem desenvolvidos para empresas } \\
\text { específicas, dificultando a generalização dos resultados. }\end{array}$ \\
\hline $\begin{array}{l}\text { Analisar } \\
\text { criticamente e } \\
\text { avaliar todos os } \\
\text { estudos; }\end{array}$ & $\begin{array}{l}\text { Os modelos para o DPS foram organizados e comparados em conformidade a estrutura } \\
\text { de nove fases do consolidado modelo para a gestão do Processo de Desenvolvimento de } \\
\text { Produtos (PDP) de Rozenfeld et al. (2006), as fases são: Planejamento Estratégico, } \\
\text { Planejamento do Projeto, Projeto Informacional, Projeto Conceitual, Projeto Detalhado, } \\
\text { Preparação da Produção, Lançamento, Acompanhamento no Mercado e Descontinuação. } \\
\text { Optou-se sobre a perspectiva da área de PDP, tendo em vista que é uma área de maior } \\
\text { volume de estudos no escopo nacional, portanto traçar um paralelo dos conteúdos } \\
\text { permite compreendê-los mais facilmente. }\end{array}$ \\
\hline $\begin{array}{l}\text { Preparar um } \\
\text { resumo crítico } \\
\text { sintetizando as } \\
\text { informações; }\end{array}$ & $\begin{array}{l}\text { Na revisão de literatura foi elaborado uma figura (Figura 1) comparativa dos catorze } \\
\text { modelos avaliados em profundidade, permitindo gerar uma caracterização geral das } \\
\text { unidades de análise, identificando principais países, grupos de estudos e setores que } \\
\text { estão preocupados com a sistematização do PDS. A figura também permite identificar } \\
\text { macrofases de alguns modelos e traçar paralelos entre as fases dos modelos estudados. }\end{array}$ \\
\hline $\begin{array}{l}\text { Apresentar } \\
\text { conclusão } \\
\text { informando a } \\
\text { evidência. }\end{array}$ & $\begin{array}{l}\text { As informações dos catorze trabalhos foram sintetizadas engendrando um modelo } \\
\text { mínimo para o PDS. O modelo mínimo visa valorizar as principais inovações trazidas } \\
\text { por cada autor, mas também prezar as repetibilidade de informações entre os estudos - os } \\
\text { consensos. Foi elaborado um quadro resumo (Quadro 2) das entradas e saídas das fases } \\
\text { do PDS, para facilitar a compreensão dos resultados. Além das fases foram destacadas } \\
\text { no quadro resumo oito atividades-chave, quatro constituintes da fase de Planejamento } \\
\text { Estratégico e quatro da fase de Projeto Detalhado. Optou-se em destacar estas atividades } \\
\text { tendo em vista que possuíam responsabilidades diferenciadas quanto às dimensões da } \\
\text { qualidade em serviços. }\end{array}$ \\
\hline
\end{tabular}

Figura 1 - Estrutura da Revisão Sistemática

\section{COMPARATIVO ENTRE OS MODELOS PARA O PDS}

Para realização de uma comparação entre modelos para o PDS utilizaram-se os catorze modelos selecionados na revisão sistemática. O resultado desta comparação fase a fase é apresentado na Figura 2, na qual os modelos são organizados em ordem cronológica de criação no eixo das abscissas e em ordem cronológica de ocorrência das fases no eixo das ordenadas. Sendo que no eixo das ordenadas, também estão distribuídos de acordo com o escopo das nove fases do modelo genérico de PDP de Rozenfeld et al (2006). Para os modelos que possuem 
macrofases, estas foram dispostas a esquerda do modelo, com as fases pertencentes a cada uma, diferenciadas intercalando caixas retangulares pontilhadas e não pontilhadas. Apenas a metade dos modelos apresentados, possui a organização de macrofases.

Nota-se uma distribuição equilibrada na quantidade de modelos nas três últimas décadas retratadas, sendo seis da década de 80, quatro da década de 90 e quatro pós anos 2000. Também pode se observar que a metade de todos os modelos avaliados são americanos. Apesar do modelo de Shostack (1984) ter sido o primeiro para a área de estudo, o modelo de Scheuing e Johnson (1989) é o mais referenciado por autores ainda hoje, uma das razões para seu reconhecimento é o seu maior nível de detalhamento, compondo-se de quinze fases. A média de fase das unidades de modelos amostradas é de dez fases. O setor financeiro e de saúde foram os primeiros a demandar maior rigor no PDS e, portanto, modelos foram desenvolvidos para esta necessidade; como Donnelly et al (1985) e Alam e Perry (2002) para o setor financeiro, e Bowers (1987) e Ramaswamy (1996) para o setor de saúde.

Fitzsimmons e Fitzsimmons (2000) desenvolveram uma classificação para os modelos de referência do PDS, com três categorias: (i) parciais, consistem de modelos somente de elementos que completam o processo ou relacionados com etapas específicas; (ii) convergentes, baseados ou originados das etapas do modelo de PDP proposto pela consultoria de Booz, Allen e Hamilton em 1982 e (iii) completos, apresentam uma visão holística do PDS. A discussão de métodos e modelos para PDP indicam a falta de capacidade de alguns deles para apoiar certos aspectos do desenvolvimento de serviços, porém quando complementados por questões específicas de serviços, são úteis para o PDS (BITRAN; PEDROSA, 1998). De fato, muitos dos modelos de PDS são classificados como convergentes, como por exemplo, Scheuing e Johnson (1989) e Bitran e Pedrosa (1998). Portanto, apesar dos modelos de serviços possuírem normalmente maior número de fases do que os modelos de produtos, eles não possuem diferenciação quanto à dinâmica de organização das informações. 


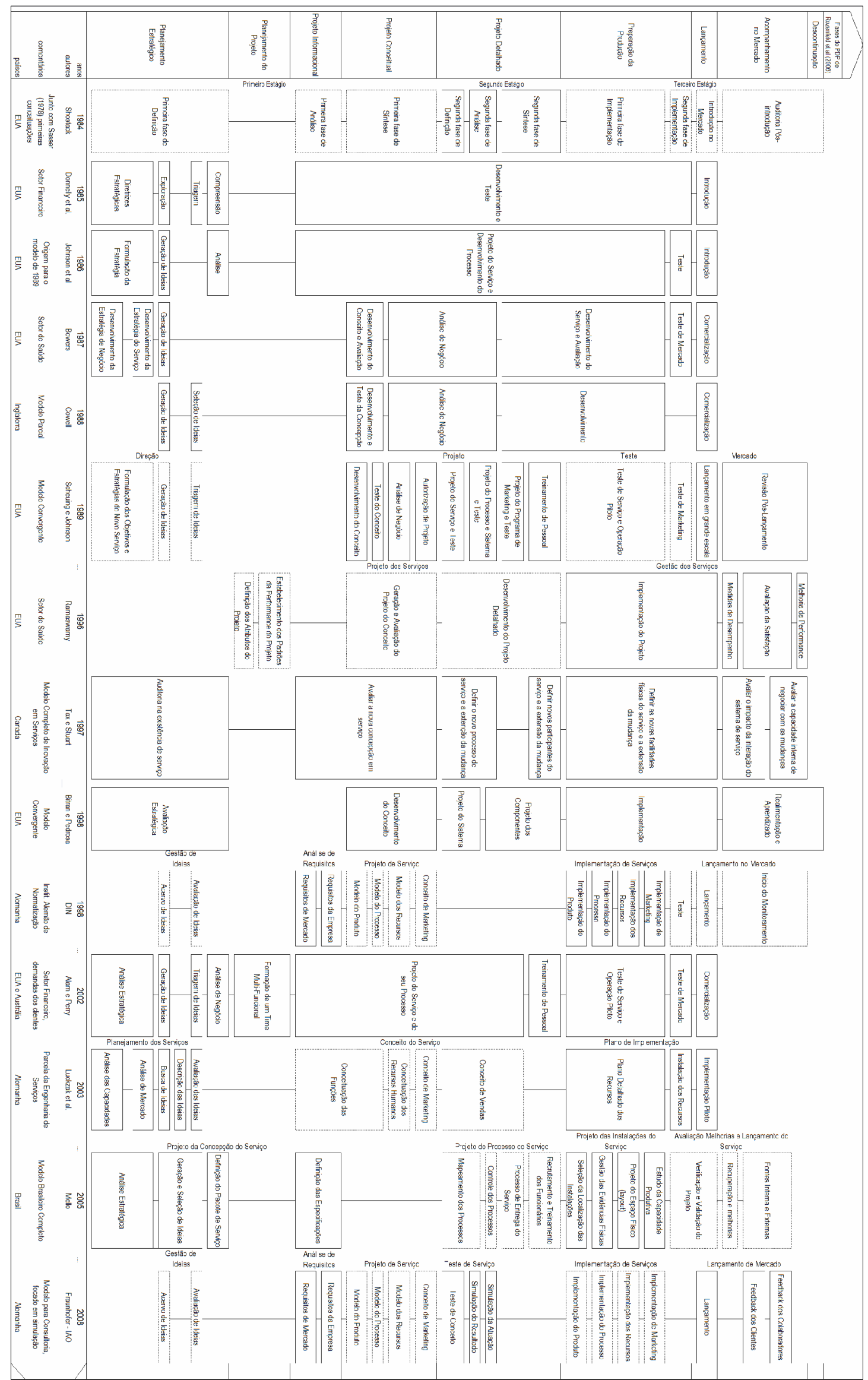

Figura 2 - Modelos da Literatura para o PDS. 
Segundo Bowers (1987) e Scheuing e Johnson (1989) os modelos faseados para o PDS podem ser reduzidos a sequência de:

(i) entender as necessidades dos consumidores;

(ii) transformá-las em conceito de serviços; conceito.

(iii) planejar o processo, estrutura, suporte e infraestrutura para este novo

De uma maneira geral, os modelos carecem de uma visão de Planejamento do Projeto na forma como é estruturada no PDP. A geração e triagem de ideias aparecem como fases nos modelos, diferentemente do PDP onde são, normalmente, tratados como atividades. Além disso, poucos modelos para o PDS tratam de uma fase específica para o Projeto Informacional e Acompanhamento no Mercado e nenhum dos modelos sugere uma fase para a Descontinuação.

Quanto a fase de Preparação da Produção, apenas no final dos anos 90 ela passou a ter suas atividades discriminada em fases independentes para alguns modelos de PDS, como nos modelos alemães DIN (1998) e Fraunhofer (2008). Esses dois modelos são praticamente iguais quanto as suas fases, uma vez que o modelo DIN (1998) está vigente na normatização alemã, por esta razão o Instituto Fraunhofer, que presta consultorias empresariais, acaba usando-o como base.

\section{RESULTADOS E DISCUSSÕES}

Nesta seção foi analisada em profundidade as nove fases genéricas em que os modelos para o PDS foram comparados na Figura 2, identificando as principais contribuições dos autores para serem incorporadas ao modelo mínimo para o PDS, apresentado ao final seção.

A primeira fase genérica é o Planejamento Estratégico. Esse contém as definições estratégicas quanto aos serviços, definindo o foco e priorizando alguns poucos critérios competitivos (dimensões da qualidade) em conformidade com a tipologia do serviço de acordo com o seu volume e variedade (gradualmente aumentando através dos níveis de serviços profissionais, lojas de serviços e serviços de massa) e porte da empresa. Para tomar decisões frente aos critérios competitivos são importantes as definições frente à segmentação e ao posicionamento no mercado (GIANESI; CORRÊA, 1994). A segmentação é a definição de grupos de consumidores com características comuns, identificadas com base em dados demográficos e psicográficos. O posicionamento é mais abrangente e envolve: identificar as necessidades dos clientes, segmentar, mapear segmentos de acordo com as necessidades e atratividade, estimar a capacidade dos concorrentes e identificar nichos e oportunidades.

Segundo Papastathopolou et al (2001) é preciso a identificação dos papéis estratégicos, a revisão do desempenho histórico, a especificação das categorias de pesquisa a serem exploradas e a distribuição de tarefas individuais e responsabilidades. Fraunhofer (2008) sugere uma estruturação do portfólio e análise do catálogo sobre os serviços oferecidos, padronização e modularização dos serviços e estratégias para internacionalizar os serviços. Tax e Stuart (1997) 
salientam a importância nessa fase de primeiramente compreender o papel dos consumidores e os efetivos benefícios que estes esperam.

Faz parte desta fase o que a norma DIN (1998) conceitua de gestão de ideias e muitos modelos descrevem esta como fase. Trata da geração e seleção de ideias para o serviço. Portanto, envolve tanto a equipe de desenvolvimento, em especial os colaboradores da linha de frente, quanto os usuários para formular o problema e as possíveis soluções. Grupos focados e entrevistas são importantes meios para levantar ideias. Segundo Mello (2005) os principais critérios para seleção de ideias são: as considerações de mercado, os planos estratégicos, as decisões gerenciais e a análise da concorrência.

$\mathrm{Na}$ fase de Planejamento do Projeto, na literatura de desenvolvimento de produtos, as informações são documentadas e inicia-se o gerenciamento do novo produto por um método de projeto, como o guia $\mathrm{PMBOK} 囚$, sendo a aprovação do Plano do Projeto o resultado desta fase (ROZENFELD et al, 2006). Devido à menor maturidade dos modelos de PDS frente aos de PDP o escopo de gerenciamento de projetos não é detalhado. O modelo de dez fases de Alam e Perry (2002) tratam de isolar a formação de um time multifuncional como uma fase, que trata da questão de seleção de recursos humanos $\mathrm{e}$ as atividades de responsabilidade de cada colaborador dentro do novo projeto determinadas em reuniões do time. A nomenclatura de 'projeto' é adotada por Ramaswamy (1996), que foca-se na preocupação de mensurar o desempenho do projeto para garantir a qualidade do serviço que será entregue ao cliente. O projeto torna-se um indicador da estabilidade e da reprodutibilidade do desempenho do serviço.

A fase de Projeto Informacional, traduzida da linguagem de processo de desenvolvimento de produtos, responsabiliza-se pela definição das especificações (atributos) do serviço, por meio do levantamento dos requisitos dos clientes. A escolha das especificações depende do grau de envolvimento dos clientes com a empresa (SWAN et al, 2002). Além das declarações técnicas, as declarações que exprimem necessidades ou expectativas dos clientes devem corresponder a padrões de projetos (RAMASWAMY, 1996). A análise dos requisitos dos clientes deve ser mensurável e verificável para relacioná-los a uma nova oportunidade de serviço. Precisam-se determinar, além dos requisitos do mercado, as perspectivas da própria empresa, formulando requisitos claros e não ambíguos para a geração do conceito (FÄHNRICH; MEIREN, 2007). Silvestro et al (1990) denominam dois tipos de especificações para serviços as de medidas rígidas (hard) e as de medidas suaves (soft). Para traduzir a voz do cliente em especificações técnicas, a literatura sugere o uso do Quality Function Deployment - QFD. Existem diversos estudos nacionais apresentando a aplicação do QFD em serviços, como os trabalhos de Ribeiro et al (2001) e Magnago e Danilevicz (2009).

No Projeto Conceitual são definidas as soluções para os requisitos levantados. A estrutura funcional de um serviço pode ser compreendida como as partes de seu processo. Um dos meios para obtenção das partes do processo começa com a hierarquização dos requisitos. Com os requisitos hierarquizados pode-se elaborar um gráfico de transferência como meio para deduzir destes as tarefas que irão compor a arquitetura do novo serviço. Com as tarefas inicia-se a classificação quanto a sua tipologia, e conforme cada tipologia pode-se realizar a análise de interdependência entre as tarefas, assim, definindo a estrutura das partes do processo (LUCZAK et al, 2007). 
Muitos modelos de PDS encontrados na literatura, além de determinar o processo e, em seguida, detalhar as suas características (Projeto Detalhado), fazem isso de forma combinada. O fator que determinou a divisão do Projeto Conceitual e Detalhado está na intensidade das definições sobre o novo serviço. Na conceituação resulta-se apenas um modelo do que será o resultado entregue do serviço ao cliente e na fase detalhada usam-se ferramentas e simulações para determinação dos recursos e controles das rotinas. Uma declaração típica de conceito deve incluir uma descrição do problema que se espera solucionar, a razão pela qual o novo serviço vai ser oferecido e o esboço de suas características e benefícios e a justificativa para a sua compra (SCHEUING; JOHNSON, 1989).

No Projeto Detalhado trata-se de descrever criteriosamente e, se possível, prototipar todo o sistema do novo serviço. As competências já existentes ou a serem desenvolvidas podem ser divididas em três tópicos bases: (i) Processos, (ii) Habilidades e Conhecimentos, e (iii) Estrutura Física (PRAHALAD; HAMEL, 1990). As ferramentas para tratar estes tópicos vêm evoluindo e buscam materializar os serviços a fim de torná-los mais compreensíveis, garantindo o sucesso póslançamento. Como é o caso do ServLab um laboratório de prototipagem rápida de serviços mantido pelo Instituto Fraunhofer, que faz simulações virtuais 3D de novos serviços. O ServLab pode testar além da estrutura física, os fluxos de processo e realizar treinamentos antes de investir em infraestrutura (FRAUNHOFER, 2008).

Quanto ao tópico de (i) Processo o mais conhecido método de definição de processo de serviços é o Service Blueprint, desenvolvido por Shostack (1984). Apesar de ter sua origem nos fluxogramas de processos industriais, é uma técnica sistemática para o mapeamento dos processos considerando o aspecto da interação com o cliente, prezando o contato front-office e as atividades back-office. O Blueprint permite a equipe enxergar o fluxo do processo e, para as partes críticas a ser entregue ao cliente, gerar um Failure Mode and Effects Analysis (FMEA) de serviços (LUCZAK et al, 2007).

Quanto ao tópico de (ii) Habilidades e Conhecimentos referem-se diretamente as capacidades dos recursos humanos que estão envolvidas. Alguns modelos de PDS salientam este tópico em uma fase em separado nomeando-a de treinamento de pessoal como Scheuing e Johnson (1989) e Alam e Perry (2002). Os prestadores do serviço (colaboradores) devem ser selecionados, treinados para as atividades do novo serviço e estruturada um meio de recompensa pelo trabalho prestado (SHOSTACK, 1987). A compreensão do papel do cliente na execução do serviço, também precisa ser considerada para definir as habilidades e conhecimentos necessários (BOWEN, 1986). Luczak et al (2007) propõem que as habilidades exigidas devem ser identificadas para cada tarefas constituinte do mapa do processo do serviço, assim permitindo compreender o perfil de competências humanas objetivado e classificar as competências específicas em: profissionais, sociais, individuais ou do sistema.

Quanto ao tópico de (iii) Estrutura Física, que trata de definição de layout, fluxos em um espaço, aquisição de um ambiente para a prestação entre outros, é um dos pontos menos tratados nos modelos de PDS (MELLO, 2005). Ramaswamy (1996) é um dos autores que trata da estrutura tangível dos serviços, mostrando que a percepção dos clientes com a qualidade pode ser influenciada por atributos como limpeza, espaço, cor, iluminação e arranjo físico do ambiente onde o serviço acontece. Mello (2005) focou-se para as questões de projeto das instalações, contudo identificou-se que estas decisões têm correspondência direta a execução do 
projeto e, portanto, os alocou na fase de Preparação da Produção. Mello (2005) sugere o uso da listagem dos estudos de Gianesi e Corrêa (1994) quanto aos critérios de fatores locacionais para escolha do melhor lugar na região, pais, área ou local específico das instalações. A escolha entre o arranjo físico por produto, processo ou posicional, os layouts mais usados em serviços (CORRÊA; CAON, 2002). A gestão de evidências físicas, como características padrões do exterior e interior do estabelecimento e todos os materiais (bens facilitadores) que serão fornecidos pelo serviço (HOFFMAN; BETESON, 2003). E, também a sugestão de estudo das capacidades produtivas dos serviços como picos de demandas, filas, eficiência, etc.

A Preparação da Produção em serviços consta com as atividades de implementação e testes dos serviços. A implementação é a etapa de execução do projeto, permitindo validar a estrutura do processo, recursos e resultado final do novo serviço. Os testes são um piloto junto ao cliente servindo para identificar a aceitabilidade e os possíveis aperfeiçoamentos tanto do novo serviço como sobre o programa de marketing para esse (SCHEUING; JOHNSON, 1989). Em paralelo, a estrutura do processo também é testada, permitindo uma comparação da situação esperada com o real e permitindo a documentação dos procedimentos operacionais a serem seguidos (BITRAN; PEDROSA, 1998).

O Lançamento, também é chamado de fase de Introdução ou Comercialização pela pelos modelos de PDS. Esta fase trata do gerenciamento das atividades de prestação do serviço em larga escala, colocando sobre acesso de todo o mercado. O momento do lançamento requer um plano com decisões básicas sobre em que época realizar, onde, para quem e como promover esse primeiro contato da empresa no mercado de acordo com a estratégia da empresa (COWELL, 1988).

O Acompanhamento no Mercado é uma fase que consta com todas as atividades de controle do desempenho do serviço pós-lançamento. Verificando se as estimativas de retorno financeiro estão correspondendo às expectativas e se a satisfação quanto à qualidade para os clientes estão sendo atendidas, por exemplo, usando respectivamente técnicas de análise financeira e o ServQual, com informações coletadas por meio de painéis, questionários e observação dos clientes. A necessidade de um constante controle da satisfação é essencial, uma vez que as expectativas dos clientes podem mudar após suas experiências com as operações do serviço ou os concorrentes podem estipular novos patamares para a qualidade, criando novos níveis mínimos de desempenhos aceitáveis (RAMASWAMY, 1996). São sugeridos nesta fase, quando necessário, planos de recuperação dos serviços para impulsionar melhorias e não simplesmente pacificar clientes insatisfeitos. A recuperação envolve o manejo de práticas de resolução de problemas (MELLO, 2005).

A fase de Descontinuação, que no escopo para produtos sugere ações para a retirada do mesmo do mercado, não é apresentada pelos modelos de PDS. Contudo, os autores compreendendo que todo serviço possui um final de ciclo de vida e, neste sentido, antes da empresa deixar de oferecê-lo propõem-se encontrar oportunidades de melhorias ou atualizações dos serviços já oferecidos ou a geração de ideias para futuros lançamento.

Todas as fases genéricas, destacando oito atividades-chave, foram incorporadas no modelo mínimo para o PDS. Para resumir o conteúdo desta subseção foi desenvolvida a Figura 3, que sintetiza entrada e saída de cada fase e atividades-chave do modelo mínimo. 


\begin{tabular}{|c|c|c|c|}
\hline \multicolumn{2}{|c|}{ Fase/Atividades do PDS } & Entrada & Saída \\
\hline \multirow{5}{*}{1} & \multicolumn{3}{|l|}{ Planejamento Estratégico } \\
\hline & $\begin{array}{l}\text { Definição dos Critérios } \\
\text { Competitivos }\end{array}$ & $\begin{array}{l}\text { Dados de mercado e revisão do } \\
\text { planejamento estratégico. }\end{array}$ & \begin{tabular}{|l|}
$\begin{array}{l}\text { Definição de critérios competitivos } \\
\text { (dimensões da qualidade). }\end{array}$ \\
\end{tabular} \\
\hline & Definições de Segmentação & $\begin{array}{l}\text { Dados demográficos e } \\
\text { psicográficos de clientes. }\end{array}$ & $\begin{array}{l}\text { Definição de grupos de consumidores } \\
\text { com características comuns. }\end{array}$ \\
\hline & $\begin{array}{l}\text { Definição do Posicionamento } \\
\text { no Mercado }\end{array}$ & $\begin{array}{l}\text { Segmentação de clientes e } \\
\text { oportunidades de mercado. }\end{array}$ & $\begin{array}{l}\text { Definição de nicho de mercado e } \\
\text { estratégias competitivas. }\end{array}$ \\
\hline & $\begin{array}{l}\text { Gestão de Ideias (gestão de } \\
\text { portfólio) }\end{array}$ & $\begin{array}{l}\text { Pesquisas com stakeholders e } \\
\text { métodos de geração de ideias. }\end{array}$ & $\begin{array}{l}\text { Levantamento de ideias preliminares } \\
\text { de serviços para serem projetados. }\end{array}$ \\
\hline 2 & Planejamento do Projeto & $\begin{array}{l}\text { Minuta de projeto do novo } \\
\text { serviço. }\end{array}$ & $\begin{array}{l}\text { Aprovação do plano de projeto, com } \\
\text { cronogramas e orçamentos. }\end{array}$ \\
\hline 3 & Projeto Informacional & $\begin{array}{l}\text { Levantamento da voz do cliente } \\
\text { clientes e informações da equipe. }\end{array}$ & $\begin{array}{l}\text { Definição e categorização dos } \\
\text { requisitos. }\end{array}$ \\
\hline 4 & Projeto Conceitual & Requisitos balanceados. & $\begin{array}{l}\text { Aprovação de um conceito (modelo) } \\
\text { das características do processo. }\end{array}$ \\
\hline \multirow{5}{*}{5} & \multicolumn{3}{|l|}{ Projeto Detalhado } \\
\hline & Definições de Processo & Conceito de serviço. & Fluxograma do processo do serviço. \\
\hline & $\begin{array}{l}\text { Definições de Habilidades e } \\
\text { Conhecimentos }\end{array}$ & $\begin{array}{l}\text { Critérios competitivos e } \\
\text { características do processo. }\end{array}$ & $\begin{array}{l}\text { Definição do perfil dos recursos } \\
\text { humanos provedores do serviço. }\end{array}$ \\
\hline & $\begin{array}{l}\text { Definições de Estrutura } \\
\text { Física }\end{array}$ & $\begin{array}{l}\text { Critérios competitivos e } \\
\text { características do processo. }\end{array}$ & $\begin{array}{l}\text { Definição da estrutura física do } \\
\text { serviço e bens facilitadores. }\end{array}$ \\
\hline & Prototipagem (simulação) & $\begin{array}{l}\text { Definições de processo, recursos } \\
\text { humanos e estrutura física. }\end{array}$ & $\begin{array}{l}\text { Homologação dos recursos } \\
\text { necessários. }\end{array}$ \\
\hline 6 & Preparação da Produção & Definições do projeto detalhado. & $\begin{array}{l}\text { Certificação e aprovação do teste } \\
\text { piloto com clientes e recursos finais. }\end{array}$ \\
\hline 7 & Lançamento & Aprovação do piloto. & Lançamento do serviço. \\
\hline 8 & $\begin{array}{l}\text { Acompanhamento no } \\
\text { Mercado }\end{array}$ & Indicadores de desempenho. & Planos de melhorias. \\
\hline 9 & Descontinuação & Análise do ciclo de vida. & Retirar serviço de operação. \\
\hline
\end{tabular}

Figura 3 - Modelo Mínimo para o PDS.

\section{CONCLUSÕES}

O modelo de características mínimas proposto visa padronizar a linguagem, consolidar atividades essenciais e facilitar o entendimento do PDS; servindo de apoio para as empresas que visam simplificar a sistematização do desenvolvimento de seus serviços ou orientar as empresas que não possuem nenhuma estrutura para este processo. Sugere-se para as empresas que visam a simplificação do PDS uma análise comparativa do seu modelo atual com o modelo de fases deste trabalho, identificando suas semelhanças e diferenças. O modelo mínimo também visa desmistificar para os gestores de serviços a dificuldade de uniformização de seu processo de desenvolvimento e cobrir parcialmente a lacuna de pesquisa de carência de estudos sobre o PDS na mesma perspectiva do PDP.

Recomendam-se como oportunidades de trabalhos futuros a elaboração de um plano de certificação para o PDS, como vem ocorrendo com o PDP, no qual existem padrões mínimos para certificação do processo, exigidos pela ISO, ou por 
regulamentações de empresa-cliente ou para atuação em um setor industrial específico. A certificação para PDS ganha apoio no contexto de serviços terceirizados, para o qual seria uma forma da empresa-contratante conhecer as capacidades e sistematização da fornecedora quanto ao PDS. Também se recomenda o desenvolvimento de trabalhos futuros que elaborem uma exemplificação didática, aplicando passo-a-passo, do modelo mínimo de PDS proposto.

\section{REFERÊNCIAS}

AKOBENG, A. K. Understanding systematic reviews and meta-analysis. Archives Disease Childhood, v. 90, p. 845-848, 2005.

ALAM, I.; PERRY, C. A customer-oriented new service development process. Journal of Services Marketing, v. 16, p. 515-34, 2002.

BEHARA, R. S.; CHASE, R. B. Service quality deployment: quality service by design. In: Sarin, R. V. (org.), Perspectives in operations management: essays in honor of Elwood S. Buffa Kluwer Academic Publisher, Norwell, MA, p. 87-99, 1993.

BITRAN, G.; PEDROSA, L. A. Structured Product Development Perspective For Service Operations. European Management Journal, v. 16, n. 2, p. 169-189, 1998.

BOOZ; ALLEN; HAMILTON; New Products Management for the 1980s. New York, 1982.

BOWERS, M. R. An exploration into new service development: process, structure and organization. Ph.D. Dissertation, Texas A\&M University, College Station. Texas, 1985.

BOWERS, M. R. Developing new services for hospitals: a suggested model. Journal of Health Care Marketing, v. 7, n. 2, 1987.

COOPER, R. G.; DE BRENTANI, U. New industrial financial services: what distinguishes the winners. Journal of Product Innovation Management, v. 8, n. 1, p. 75-90, 1991.

CORRÊA, H. L.; CAON, M. Gestão de Serviços. São Paulo: Atlas, 2002.

COWEL, D. W. New service development. Journal of Marketing Management, v. 3, n. 3, p. 296-312, 1988.

D'AVENI, R. A. Hypercompetition: managing the dynamics of strategic maneuvering. New York: Free Press, 1994.

DESZCA, G.; MUNRO, H.; NOORI, H. Developing breakthrough products: challenges and options for market assessment. Journal of Operations Management, v. 17, p. 613-630, 1999. 
DEUTSCHES INSTITUT FÜR NORMUNG (DIN). Service Engineering: entwicklungsbegleitende Normung (EBN) für Dienstleistungen. Berlin: 1998.

DOMHOLDT, E. Rehabilitation research: principles and applications. Missouri: Elsevier Saunders, 2005.

DONNELLY, J. H.; BERRY, L. L.; THOMPSON, T. W. Marketing financial services: a strategic vision. Homewood: Dow Jones-Irwin, 1985.

EASINGWOOD, C. J. New product development for service companies. Journal of Product Innovation Management, v. 3, n. 4, p. 264-75, 1986.

EDGETT, S. J. Developing new financial services within UK building societies. International Journal of Bank Marketing, v. 11, n. 3, p. 35-43, 1993.

FÄHNRICH, K. P.; MEIREN, T. Service engineering: state of the art and future trends, In: Spath, D.; Fähnrich, K.P. (org.): Advances in Services Innovations. Springer: Berlin, p.3-16, 2007.

FITZSIMMONS, J. A.; FITZSIMMONS, M. J. New service development - creating memorable experiences. Sage Publications: Thousand Oaks, p. 1-32, 2000.

FITZSIMMONS, J. A.; FITZSIMMONS M. J. Administração de Serviços: operações, estratégias e tecnologia da informação. 4 ed. Porto Alegre: Bookman, 2005.

FRAUNHOFER, Business Process Management Tools - IOF: Fraunhofer Institute for Industrial Engineering IAO. Disponível em: <http://www.iao.fraunhofer.de/index.php>, Acessado em: 7 de outubro de 2010, 2008.

GIANESI, I. G. N.; CORRÊA, H. L. Administração estratégica de serviços: operações para a satisfação do cliente. São Paulo: Atlas, 1994.

HOFFMAN, K. D.; BATESON, J. E. G. Princípios de marketing de serviços. Conceitos, estratégias e casos. São Paulo: Pioneira Thomson Learning, 2003.

JOHNSON, E.; SCHEUING, E. E.; GAIDA, K. A. Profitable service marketing. Homewood: Dow Jones-Irwin, 1986.

JOHNSON, S. P.; MENOR, L. J.; ROTH, A. V.; CHASE, R. B. A critical evaluation of the new service development process: integrating service innovation and service design. In: Fitzsimmons, J. A. e Fitzsimmons, M. J, New Service Developmente, p. 132, 2000.

FITZSIMMONS, M. J. New service development - creating memorable experiences. Sage Publications: Thousand Oaks, p. 1-32, 2000.

JOHNSTON, R. Service operations management: return to roots. International Journal of Operations and Production Management, v. 19, n.2, p. 104-124, 1999. 
JONG, J. P. J.; VERMEULEN, P. A. M. Organizing successful new service development: a literature review. Management Decision, v.41, n. 9, p. 844-58, 2003.

LAW, M.; PHILP, I. Systematically reviewing the evidence. In: Law M. Evidencebased rehabilitation: a guide to practice. Thorofare (NJ): SLACK Inc., 2002.

LEVITT, T. Marketing intangible products and product intangibles. Harvard Business Review, v. 59, p. 94-102, 1981.

LINDE, K.; WILLICH, S. N. How objective are systematic reviews? Differences between reviews on complementary medicine. Journal of the Royal Society of Medicine, v. 96, p. 17-22, 2003.

LUCZAK $H_{\text {.; }}$ LIESTMANN V.; GILL C. Service engineering industrieller dienstleistungen. In: Bullinger H. J.; Scheer A. W. (org.) Service Engineering: entwicklung und gestaltung innovativer dienstleistungen. Springer: Berlin Heidelberg New York, p. 443-466, 2003.

LUCZAK, H.; GILL, C.; SANDER, B. Architecture for service engineering - The design and development of industrial service work. Advances in Services Innovations, v. 1, p. 47-63, 2007.

MA, Q.; TSENG, M. M.; YEN, B. A generic model and design representation technique of service products. Technovation, n. 22, p. 15-39, 2002.

MAGEE, D. J. Systematic reviews (meta-analysis) and functional outcome measures. Aindow: Apostila, Developmental Ed, 1998.

MAGNAGO, P. F.; DANILEVICZ, A. M. F. Planejamento de melhorias nos serviços de distribuição: uma aplicação do QFD no setor de cosméticos. In: CBGDP, Anais... São José dos Campos - SP, 2009.

MARTIN, C. R.; HORNE D. A. Services innovation: successful versus unsuccessful firms. International Journal of Services Industry Management, v. 4, n. 1, p. 49-65, 1993.

MEIREN, T.; BARTH, T. Service Engineering in Unternehmen umsetzen - Leitfaden für die Entwicklung von Dienstleistungen. Stuttgart: 2002.

MELLO, C. H. P. Modelo para projeto e desenvolvimento de serviço. Tese (Doutorado em Engenharia de Produção), Universidade de São Paulo. São Paulo, 2005.

MENOR, L. J.; TATIKONDA, M. V.; SAMPSON, S. E. New service development: areas for exploitation and exploration. Journal of Operations Management, v. 20, p. 135-157, 2002. 
PAGE, A. L.; GARY, R. S. Growth and development of a body of knowledge: 16 years of new product development research, 1989-2004. Journal of Product Innovation Management, v. 25, n. 3, p. 233-248, 2008.

PAPASTATHOPOLOU, P.; AVLONITIS, G.; INDOUNAS, K. The initial stages of new service development: a case study from the Greek bank sector. Journal of Financial Services Marketing, 2001.

PARASURAMAN, A.; VALARIE, A.; ZEITHAML, P.; BERRY, L. L. A conceptual model of $S Q$ and its implications for future research. Journal of Marketing, v. 49, p. 41-50, 1985.

PRAHALAD, C. K.; HAMEL, G. The core competence of the corporation. Harvard Business Review, v. 68, n. 3, 1990.

RAMASWAMY, R. Design and management of service processes - keeping customers for life. Reading: 1996.

RIBEIRO, J. L. D.; ECHEVESTE, M. E.; DANILEVICZ, A. M. F. A utilização do QFD na otimização de produtos, processos e serviços. Série Monográfica Qualidade. Porto Alegre: FEENG/UFRGS, 2001.

ROZENFELD, H.; FORCELLINI, F. A.; AMARAL, D. C.; TOLEDO, J. C.; SILVA, S. L.; ALLIPRANDINI, D. H.; SCALICE, R. K. Gestão de desenvolvimento de produtos: uma referência para a melhoria do processo. São Paulo: Saraiva, 2006.

SAMPAIO, R. F.; MANCINI, M. C. Estudos de revisão sistemática: um guia para síntese criteriosa da evidência científica. Revista Brasileira de Fisioterapia, v. 11, n. 1, p. 83-89, 2007.

SCHEUING, E. E.; JOHNSON, E. M. A proposed model for new service development. Journal of Services Marketing, v. 3, n. 2, p. 25-35, 1989.

SHOSTACK, G. L. Designing services that deliver. Harvard Business Review, v. 1, p. 133-139, 1984.

SHOSTACK, G. L. Service positioning through structural change. Journal of Marketing, v. 51, winter, p. 33-43, 1987.

SILVESTRO, R.; JOHNSTON, R.; FITZGERALD, L.; VOSS, C. Quality measurement in service industries. International Journal of Service Industries Management, v. 1, n. 2, 1990 .

SWAN, J. E.; BOWERS, M. R.; GROVER, R. Customer involvement in the selection of service specifications. Journal of Services Marketing, v. 16, n. 1, p. 88-103, 2002.

TAX, S. S.; STUART, I. Designing and implementing new service: the challenges of integrating service systems. Journal of Retailing, New York, v. 73, n. 1, p. 105-134, winter, 1997. 